\title{
Continuous-type splenogonadal fusion: A case report
}

\author{
GUIZHEN HUANG ${ }^{1 *}$, YIDONG HUANG ${ }^{2 *}$, LI ZENG $^{2}$, MIAO YUAN $^{2}$, YANG WU ${ }^{2}$ and LUGANG HUANG ${ }^{2}$ \\ ${ }^{1}$ Department of Pediatric Surgery, The First Affiliated Hospital of Xiamen University, Xiamen, Fujian 361000; \\ ${ }^{2}$ Department of Pediatric Surgery, West China Hospital of Sichuan University, Chengdu, Sichuan 610041, P.R. China
}

Received August 16, 2015; Accepted December 23, 2016

DOI: $10.3892 /$ etm.2017.4198

\begin{abstract}
Splenogonadal fusion (SGF) is a rare congenital malformation. Since it lacks characteristic features, very few cases of SGF have been diagnosed preoperatively. Laparoscopy was effective in both diagnosing and surgically treating this condition. Herein, we reported left side SGF in a male patient who was diagnosed during laparoscopic exploration, and Fowler-Stephens orchidopexy was implemented at the same time. The patient was followed up for one year. At a 6-month follow-up, the left scrotum demonstrated swelling and the internal contents were hard. An ultrasound of this testicle indicated non-uniform, splenic-like organization. However, at the one-year follow-up, the volume of splenic-like organization was reduced but the testicular size did not exhibit further atrophy.
\end{abstract}

\section{Introduction}

Splenogonadal fusion (SGF) is a rare congenital malformation. The condition was first described in 1883, but fewer than 200 cases have been reported to date (1). Only a few cases have been diagnosed preoperatively, which were typically regarded as cryptorchidism, testicular tumors or inguinal hernia, and accidentally determined to be SGF after exposure of the inguinal canal during surgery (2). This disease commonly leads to a preoperative misdiagnosis of testicular cancer, causing testes to be mistakenly removed in nearly a third of SGF cases $(3,4)$. In order to avoid these errors, increased awareness of the diagnosis of and treatment for SGF therefore is required. In this case report, we summarize our experiences of treating SGF and review the literature to improve the understanding of this disease and provide helpful suggestions for future treatment strategies.

Correspondence to: Dr Lugang Huang, Department of Pediatric Surgery, West China Hospital of Sichuan University, 37 Guoxue Alley, Chengdu, Sichuan 610041, P.R. China

E-mail: lugang992001@aliyun.com

${ }^{*}$ Contributed equally

Key words: splenogonadal fusion, splenogonadal fusion, case report

\section{Case report}

A 4-year-old boy presented to the Department of Pediatric Surgery, West China Hospital of Sichuan University (Chengdu, China) with bilateral undescended testes from birth in April 2013. Physical examination indicated that the bilateral scrota were empty, the testes were also not present in the inguinal region, and the patient's penis was normal. An ultrasound confirmed that there were no testicular masses in the bilateral scrota and inguinal regions. In accordance with the above clinical findings, the patient was diagnosed with bilateral cryptorchidism, and therefore laparoscopic gonad exploration was performed. Complete abdominal exploration revealed gonad-like tissue connected with a smooth, fleshy, brown, cord-like structure beside the left colon, but left spermatic cord blood vessels were not observed. The cord-like structure was connected to the spleen (which was normal), and was approximately $5-6 \mathrm{~cm}$ long and $1.5 \mathrm{~cm}$ in diameter (Fig. 1A and B). The spleen cord was cut off and the gonad-like tissues were pulled down into the scrotum through the inguinal canal (Fig. 1C-E). A small incision was made in the scrotum skin to expose the gonad-like tissue. A small piece of gonad-like tissue was harvested for pathological examination. The result of pathological examination demonstrated that the gonad-like tissue was a normal testis. As a result, the spleen cord was resected and Fowler-Stephens orchidopexy was implemented. The right testicle was located in the right iliac fossa, $2 \mathrm{~cm}$ from the right deep inguinal ring. The right spermatic cord was short and the testis exhibited dysplasia. The right testicle was moved posteriorly and fixed by single-stage laparoscopic Fowler-Stephens orchidopexy (Fig. 1F). Postoperatively, the patient recovered well. At a 6-month follow-up in the Outpatient Department of Pediatric Surgery, West China Hospital of Sichuan University physical examination revealed that the right testis was normal; however, the left scrotum demonstrated swelling and the internal contents were hard. An ultrasound of this testicle indicated non-uniform, splenic-like organization, with abundant blood flow and a reduction in the testicular size (Fig. 2). We will continue to follow-up this patient; if hyperplasia of the splenic tissue in the scrotum is detected during a subsequent follow-up, a reoperation will be performed. At one-year follow-up, the volume of splenic-like organization was reduced but the testicular size did not exhibit further atrophy, thus reoperation has not been performed. We will continue to follow-up this patient. 

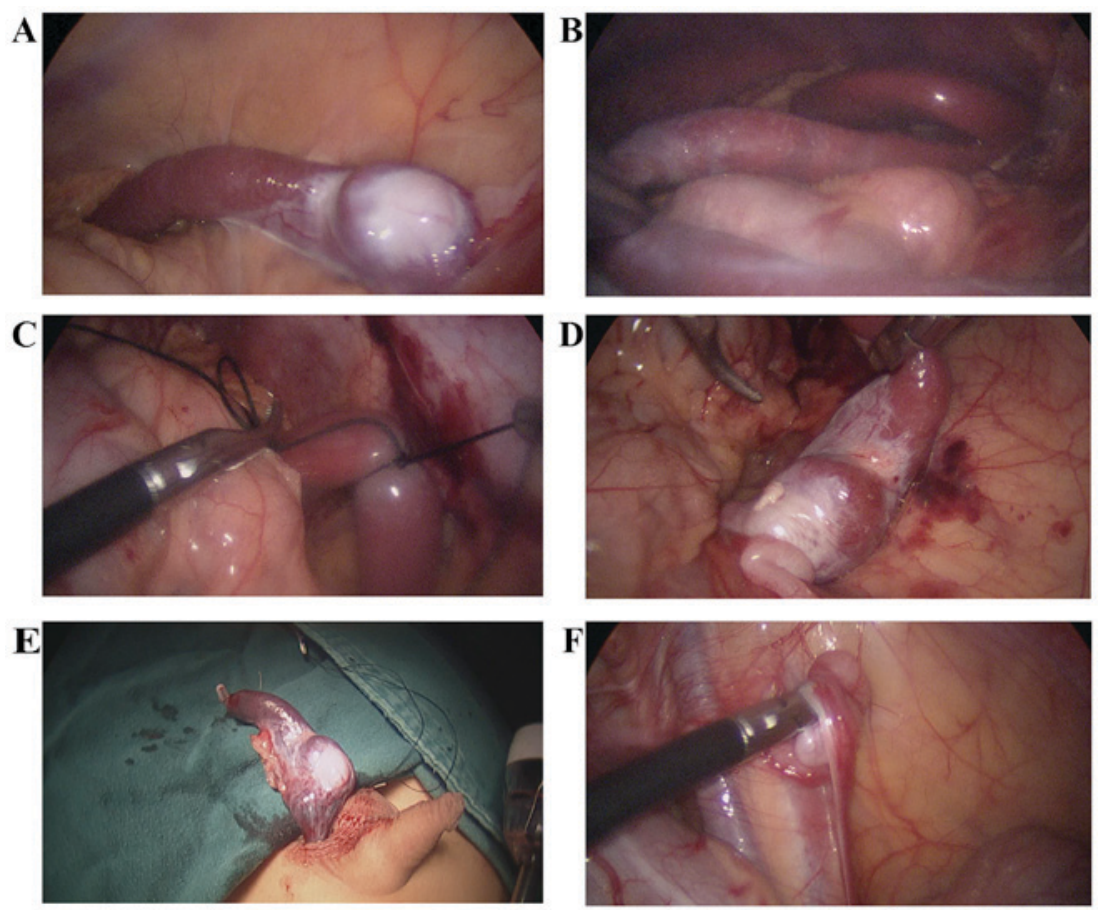

Figure 1. Perioperative photograph of the splenic cord joined to the testis, confirming the diagnosis of continuous type splenogonadal fusion. (A) A cylindrical mass beside the left colon, with the testic at the distal end of this; and (B) continuation of this mass into the spleen. (C) During laparoscopy, the left testicle was identified $3 \mathrm{~cm}$ from the spleen. (D) Spermatic cord and vas deferens dysplasia. (E) The left testicle was descended posteriorly and fixed by single-stage laparoscopic Fowler-Stephens orchiopexy; and (F) the right testicle was located in the right iliac fossa, $2 \mathrm{~cm}$ from the right deep inguinal ring.
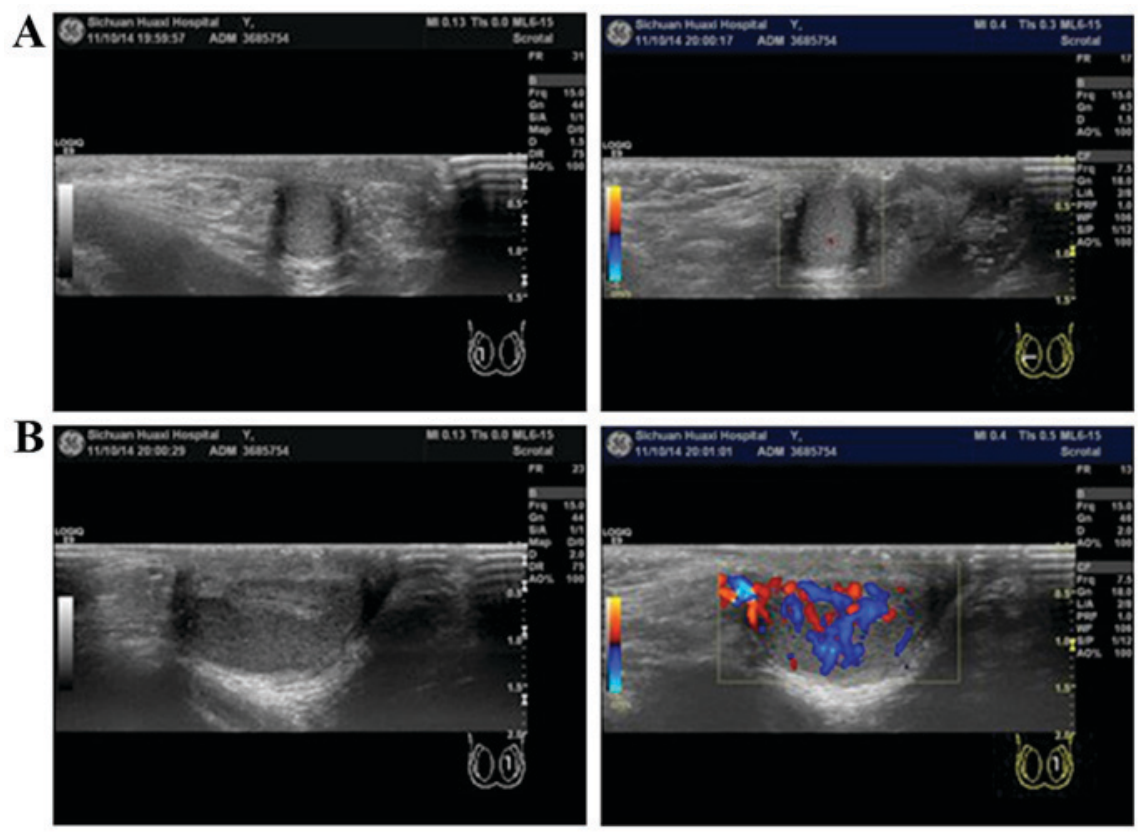

Figure 2. Ultrasound findings of the scrotum after 6 postoperative months. (A) Right and (B) left scrota. Blue and red indicate the tissue blood supply; blue indicates blood deviates from the ultrasound probe and red indicates blood that flows to the ultrasound probe.

\section{Discussion}

SGF is a rare congenital malformation, which was first reported in 1883 by Bostroem $(1,3)$ and Pommer made a detailed report in 1889 (5). However, there have been fewer than 200 cases worldwide since the disease was first described (1). With regard to diagnoses, $70 \%$ of patients are $<20$ years old when diagnosed and $50 \%$ are $<10$ years old. There are more male than female cases, at a ratio of 16.6:1 (6). In 1956, Putschar and Manion (7) divided the disease into two types: Continuous and discontinuous. Continuous type indicates a cord-like tissue (spleen tissue or fibrous tissue) directly connecting the gonad and spleen, whereas discontinuous type indicates gonad tissue fused with ectopic accessory spleen but there is no connection between the ectopic accessory spleen and principal spleen $(1,2,8)$. The present case is an example of continuous 
SGF. Previous studies indicate that the frequency of the two types is equal (5), but other studies suggest that the frequency of discontinuous type SGF was lower than that of continuous $\operatorname{SGF}(2,8,9)$.

The exact cause of SGF remains unknown, but it is generally believed to occur prior to gonad decline, between 5-8 weeks of pregnancy (10). During embryogenesis, the epithelial cells of the body cavity and the intestinal cells form the spleen in the dorsal intestine. Simultaneously, during rotation, the spleen is close to the gonads. The rotation process may therefore lead to fusion of the two. The gonads begin to decline at 8 weeks after gestation. If the gonad and spleen are fused at this time, the partial splenic tissue adhered to the gonad would descend with the gonads, so the splenic tissue may appear in any location of the primordial gonad's descent path, even in the inguinal canal or within the scrotum $(8,10,11)$.

Almost all previous cases of SGF (98\%) occurred on the left side (2), often accompanied by left cryptorchidism, short limb malformation and a small jaw. In addition, it may also present as bilateral cryptorchidism, hypospadias, anal malformation and diaphragmatic hernia $(8,9,11)$. Complications occurring in continuous type SGF are 5 times more frequent than in discontinuous type SGF (8). Only a few cases have been diagnosed preoperatively, usually due to cryptorchidism, testicular tumors or inguinal hernia (4); most are reported following exposure of the inguinal canal during surgical treatment $(2,3,11)$. According to a previous study, approximately $30 \%$ of cases reporting scrotal swelling are misdiagnosed as testicular tumors and result in unnecessary orchiectomy (3). A few cases have presented as emergencies, with symptoms such as orchitis and testicular torsion or trauma. Hines and Eggum (12) reported a case of intestinal obstruction caused by the pressure of the splenic tissue in a continuous type SGF patient. However, there have also been a few cases diagnosed preoperatively by ultrasound, computed tomography or magnetic resonance imaging $(6,9,13)$. Most cases of SGF reported by such imaging examination were continuous SGF, demonstrating a tubular structure fused with the testicles $(8,9,11)$. In the current case, the patient presented with bilateral cryptorchidism but with no other abnormalities. A clear diagnosis was not made prior to surgery. Perioperative frozen pathological examination aids exclusion of the possibility of testicular tumors and avoiding orchiectomy because of misdiagnosis.

Following the development of laparoscopic techniques, an increase of SGF diagnosis is expected. For children with cryptorchidism, if laparoscopic exploration does not indicate the presence of testicular tissue in the deep inguinal ring and iliac fossa, the paracolic sulci should be investigated to prevent misdiagnosis.

When SGF diagnosis is apparent but asymptomatic, the necessity of surgery is controversial. SGF may not increase the risk of testicular cancer, but SGF often associates with cryptorchidism, a risk factor for testicular cancer; as a result, SGF indirectly increases the risk of testicular tumors $(2,4)$. To date only 4 cases of patients with SGF and testicular tumors have been reported, and all of these presented with merged cryptorchidism (2). Therefore, for patients with combined cryptorchidism, testicular descent fixation appears to be the most appropriate therapeutic option. There is disagreement as to whether the splenic tissue adhering to the testis should be completely removed or not. Our team of surgeons hold the belief that the tissue should not be completely eliminated, therefore this protocol was followed. In the present case, it was suggested that testicular blood supply would be affected if splenic tissue was completely removed, so it was retained. At a 6-month follow-up, the splenic tissue grew and the testicle became smaller. To the best of our knowledge, whether splenic tissue hyperplasia causes ipsilateral testicular atrophy is unknown. The currently described patient may be subjected to a second surgery to resect splenic tissue in the left scrotum. This has not yet occurred and the patient is still being followed-up.

In conclusion, SGF is a rare disease, presenting difficulty in diagnosis due to an absence of typical clinical symptoms. This disease should be highlighted in order to avoid the misdiagnosis of testicular tumors and performing unnecessary orchiectomies; laparoscopic exploration is therefore helpful in diagnosis and treatment of patients with high cryptorchidism, and perioperative frozen pathological examination may help eliminate differential diagnosis of testicular tumors.

\section{Acknowledgements}

This work was supported by the Sichuan Province foundation (grant no 2017SZ0060) of the Science and Technology Department of Sichuan Province.

\section{References}

1. Jayasundara JA, Vithana VH and Lamahewage AK: A case of continuous-type splenogonadal fusion. Singapore Med J 54: e123-e124, 2013

2. Lopes RI, de Medeiros MT, Arap MA, Cocuzza M, Srougi M and Hallak J: Splenogonadal fusion and testicular cancer: Case report and review of the literature. Einstein (Sao Paulo) 10: 92-95, 2012.

3. Karaman MI and Gonzales ET Jr: Splenogonadal fusion: Report of 2 cases and review of the literature. J Urol 155: 309-311, 1996.

4. Sountoulides P, Neri F, Bellocci R, Schips L and Cindolo L: Splenogonadal fusion mimicking a testis tumor. J Postgrad Med 60: 202-204, 2014

5. Carragher AM: One hundred years of splenogonadal fusion. Urology 35: 471-475, 1990.

6. Varma DR, Sirineni GR, Rao MV, Pottala KM and Mallipudi BV: Sonographic and CT features of splenogonadal fusion. Pediatr Radiol 37: 916-919, 2007.

7. Putschar WG and Manion WC: Splenicgonadal fusion. Am J Pathol 32: 15-33, 1956.

8. Bosnalı O, Cici İ, Moralığlu S and Cerrah-Celayir A: Continuous-typ esplenogonadal fusion: Report of a rare case. Turk J Pediatr 56: 680-683, 2014.

9. Keyik B, Yanik B, Conkbayir I, Tuygun C, Kizilgoz V and Hekimoğlu B: Continuous-type splenogonadal fusion associated with an ipsilateral testicular atrophy: Sonographic findings. J Clin Ultrasound 38: 161-163, 2010.

10. Le Roux PJ and Heddle RM: Splenogonadal fusion: Is the accepted classification system accurate? BJU Int 85: 114-115, 2000.

11. Chiaramonte C, Siracusa F and Li Voti G: Splenogonadal fusion: A genetic disorder?-report of a case and review of the literature. Urol Case Rep 2: 67-69, 2014.

12. Hines JR and Eggum PR: Splenic-gonadal fusion causing bowel obstruction. Arch Surg 83: 887-889, 1961.

13. Croxford WC, Pfistermuller KL, Scott F and Pope AJ: Splenogonadal fusion presenting clinically and radiologically as a seminoma. Urol Case Rep 3: 204-205, 2015. 